\title{
Optical Properties of Nanocomposite Films: Size-tuned vs. Shape-tuned Silver Nanoparticles
}

\author{
Maryuri Roca*, Hannah Emily Skipper, Jessica Ranesizafiniaina Ndrianasy \\ Chemistry Department, Skidmore College, Saratoga Springs, NY 12866, United States \\ *Corresponding author: mroca@skidmore.edu
}

Received April 13, 2019; Revised May 24, 2019; Accepted June 07, 2019

\begin{abstract}
Color change is a desirable response when designing user-friendly chemical sensors. However, when preparing colored nanocomposites sensors, it can be challenging to transfer the color from solution into films. This work demonstrates that films of various colors can be prepared by blending premade nanoparticles with poly(vinyl alcohol) (PVA); however, the color in solution differed from the color in film. While, this color discrepancy and the appearance of the films depended on how the optical properties of nanoparticles were tuned, UV-visible spectroscopy and Transmission Electron Microscopy (TEM) showed that changes in nanoparticle's morphology was not the cause of the color discrepancy. Reflecting the polydispersity of nanoparticles in solution, more homogeneous colored films were obtained by controlling the size of nanoparticles rather than their shape. This work shows how to readily prepare silver-PVA nanocomposite films of various colors, which may facilitate the design of nanocomposites intended for chemical sensing based on the optical properties of silver nanoparticles.
\end{abstract}

Keywords: color, PVA, plasmonic nanocomposite, blended polymer

Cite This Article: Maryuri Roca, Hannah Emily Skipper, and Jessica Ranesizafiniaina Ndrianasy, "Optical Properties of Nanocomposite Films: Size-tuned vs. Shape-tuned Silver Nanoparticles." American Journal of Nanomaterials, vol. 7, no. 1 (2019): 22-29. doi: 10.12691/ajn-7-1-3.

\section{Introduction}

Polymeric films containing nanoparticles are nanocomposite materials with novel optical properties that can be controlled by modifying the composition of both polymer and metal nanoparticles [1,2]. While the initial integration of nanoparticles and polymers sought to improve the properties of polymers, current works focus on exploiting and even discovering new properties of those known for nanoparticles in liquid phase. Confining nanoparticles in films has gone beyond preventing the unwanted aggregation that nanoparticles experience in solution, and new nanocomposites have been engineered to control the interaction of nanoparticles [1]. However, preserving the properties and controlling the distribution of nanoparticles in films can be challenging, in most cases requiring modifications of the nanoparticles to secure its compatibility with the polymer. Silver nanoparticles are advantageous to study such compatibility because their light extinction falls in the visible spectra and changes in color offer information about the interaction between nanoparticle and polymer. Films made of silver nanoparticles embedded in poly (vinylalcohol) (PVA) are of interest because the transparency, solubility, and permeability of PVA makes this matrix attractive for sensors that use Localized Surface Plasmon Resonance (LSPR) [3,4,5] and Surface-Enhanced Raman Spectroscopy (SERS) [6,7].
The simple judgement of color is powerful in exploring the properties of nanomaterials. Tuning the morphology of silver nanoparticles to obtain different colors has been vastly studied in solution phase [8,9]. Similar morphology-controlled tuning has scarcely been done for films, and films of different colors have been prepared by controlling the refractive index of the nanocomposite, metal composition of nanoparticles, and surface modification of the nanoparticles $[10,11,12]$. Alternatively, the color of a given film can be changed by applying an external stimulus (electrical, optical, mechanical, thermal, etc.) to the nanocomposite to alter the morphology or coupling of nanoparticles $[13,14,15,16]$. While, these complex modifications have produced colored films, the simple embedding of silver nanoparticles in films generally produces a single color, yellow [17-23].

There are two main approaches to prepare PVA films with embedded silver nanoparticles. In the in-situ approach, silver ions are mixed with the polymer, and then nanoparticles are synthesized in the matrix of the polymer during or after the formation of the film using heat, radiation or chemical reagents $[21,24,25]$. The ability to create patterns in the film is a major advantage of the in-situ approach [26], but only yellow films have been obtained by tuning the size of silver nanoparticles [27]. In the ex-situ or blending approach, premade nanoparticles are added to the polymer before, during or after the formation of the film [1,2]. A major advantage of the blending approach is that nanoparticles with particular properties can be premade. However, transferring these 
properties to a film can be challenging [12,28], and an external stimulus is required to tune such properties. This manipulation can bring unwanted changes to the film itself.

To gain insight on how to control the color of silverPVA nanocomposite films, shape-tuned and size-tuned nanoparticles were compared in their feasibility to produce homogeneously colored films by contrasting the physical appearance of films, monitoring the stability of the color during the synthesis, and analyzing the morphology of nanoparticles in solutions and films. This work shows how to tune the optical properties of plasmonic nanocomposites without external stimulus using the blending approach.

\section{Materials and Methods}

\subsection{Materials}

Silver nitrate and sodium borohydride were obtained from ACROS. Trisodium citrate dihydrate, ascorbic acid, and hydrogen peroxide (30 wt \%) were obtained from Fisher Scientific. PVA (Poly (vinyl alcohol) 85,000 - 124,000 molecular weight), and PVP (Poly (vinyl pyrrolidone) 40,000 average molecular weight) were obtained from Sigma-Aldrich. Ultrapure water $(18 \mathrm{M} \Omega \cdot \mathrm{cm})$ was obtained using a NanoPure system from Barnstead. All glassware was washed with concentrated nitric acid and ultrapure water before use.

\subsection{Synthesis of Silver Nanoparticle Solutions}

Colored solutions of silver nanoparticles were prepared using two methods. The first was the vastly studied reduction of silver ion in the presence of citrate, where the shape of nanoparticles is controlled by varying the amount of borohydride [29,30]. The second was the more recent Simultaneous Multiple Asymmetric Technique (SMART), where the size of nanodisks is controlled by varying the amount of ascorbic acid [31].

In a typical synthesis of shape-tuned nanoparticles, a $15.0 \mathrm{~mL}$ mixture of $0.10 \mathrm{mM}$ silver nitrate, $1.5 \mathrm{mM}$ trisodium citrate, and $0.030 \mathrm{mM}$ PVP was vigorously stirred. A volume of $0.036 \mathrm{~mL}$ of $30 \%$ hydrogen peroxide was added just before adding $0.060 \mathrm{~mL}$ or $0.10 \mathrm{~mL}$ of $0.10 \mathrm{M}$ sodium borohydride to obtain red or blue solutions, respectively. To prepare yellow solutions the hydrogen peroxide was omitted and $0.080 \mathrm{~mL}$ of fresh $0.10 \mathrm{M}$ sodium borohydride was added. The mixture was stirred until the final color developed, approximately 30 minutes. Solutions were aged in the dark without stirring for 2 hours.

In a typical synthesis of size-tuned silver nanoparticles, a solution of $0.18 \mathrm{mM}$ silver nitrate and $0.145 \mathrm{mM}$ PVP was prepared in a volumetric flask. Aliquots of $100 \mathrm{~mL}$ were placed in $500 \mathrm{~mL}$ Erlenmeyer flasks. Working very quickly, a volume of $0.10 \mathrm{M}$ ascorbic acid was added to the silver solution, the mixture was swirled, and $0.15 \mathrm{~mL}$ of $1.0 \mathrm{mM}$ sodium borohydride was added to instantaneously develop the color. The colored solution was swirled for 10 more seconds and aged in the dark for 3 hours. Yellow, red, or blue solutions were obtained by adding $2.0 \mathrm{~mL}, 0.50 \mathrm{~mL}$ or $0.10 \mathrm{~mL}$ of ascorbic acid, respectively.
All solutions were washed and concentrated 10 fold by centrifuging $10 \mathrm{~mL}$ of solution at $14,000 \mathrm{rpm}$ for $30 \mathrm{~min}$ in a Labnet microcentrifuge. The supernatant was decanted and the pellets were dispersed by sonication. Water was added to achieve a final volume of $1 \mathrm{~mL}$.

\subsection{Preparation of Colored Films}

Colored films were prepared by entrapping nanoparticles in PVA [23]. In a typical preparation, a nanoparticle solution was mixed in a 1:1 volume ratio with $10 \%$ aqueous PVA solution, vortexed for 30 seconds, and let stand for 10 minutes. Approximately $1 \mathrm{~mL}$ of the colored PVA was dropped on an acrylic sheet and left to dry overnight at room temperature.

\subsection{Instrumentation}

Light extinction of solutions and films was measured using an Ocean Optics UV-Vis spectrophotometer with a $1-\mathrm{cm}$ cuvette and a reflectance probe, respectively. Shapes and sizes of nanoparticles were measured using a Zeiss Libra 120 Transmission Electron Microscope. For solution-phase nanoparticles, approximately $5 \mu \mathrm{L}$ of nanoparticle solution was dropped onto a 400-mesh copper grid coated with carbon/Formvar (from TedPella) and allowed to dry. For nanoparticles embedded in film, Ultramicrotomy was used to section the colored films [23]. In a typical preparation, colored film was embedded in Spurr Low Viscosity Embedding Media (from Electron Microscopy Sciences), and the embedded film was cut into $80 \mu \mathrm{m}$ thick sections using a Leika Ultracut $\mathrm{E}$ Ultramicrotome with a glass knife and boat filled with water. Sections floating on the surface of the water were collected onto a TEM copper grid and allowed to dry.

\section{Results and Discussion}

\subsection{Appearance of PVA Films Embedded with Silver Nanoparticles}

Nanoparticles were synthesized using two methods that controlled either the shape or size of nanoparticles to tune the color of the solution. PVA films colored by embedded silver nanoparticles were produced in a three-step procedure, as summarized in Figure 1. First, silver nanoparticles were cleaned and concentrated via centrifugation. Second, concentrated nanoparticles were diluted in a solution of aqueous PVA. Finally, colored films were obtained by solvent evaporation. Since the focus of this work was to examine the transferability of the color in a simple approach, the thickness of the films was not explicitly controlled.

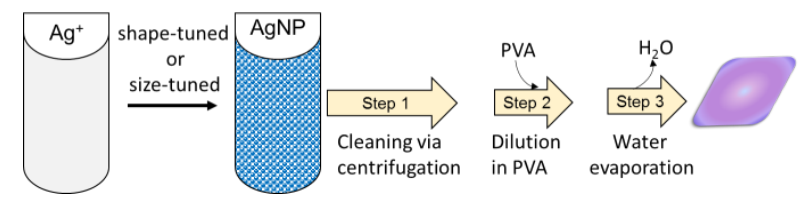

Figure 1. Experimental strategy for the preparation of PVA films colored by shape-tuned or size-tuned silver nanoparticles 

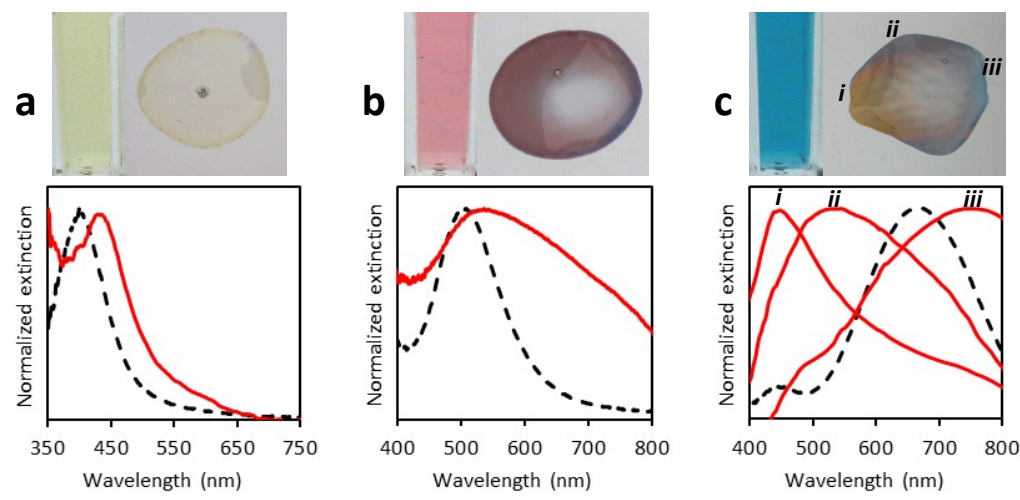

Figure 2. Optical characteristics of solutions and films of silver nanoparticles tuned by shape. Top are the photographs of (a) yellow, (b) red, and (c) blue solutions and films. The bottom are the normalized extinction spectra of (dashed black line) solutions and (solid red line) different colored zones in the films. The zones in the films are labeled to match the spectra as $i, i i$, and $i i i$ for yellow, purple and blue zones, respectively
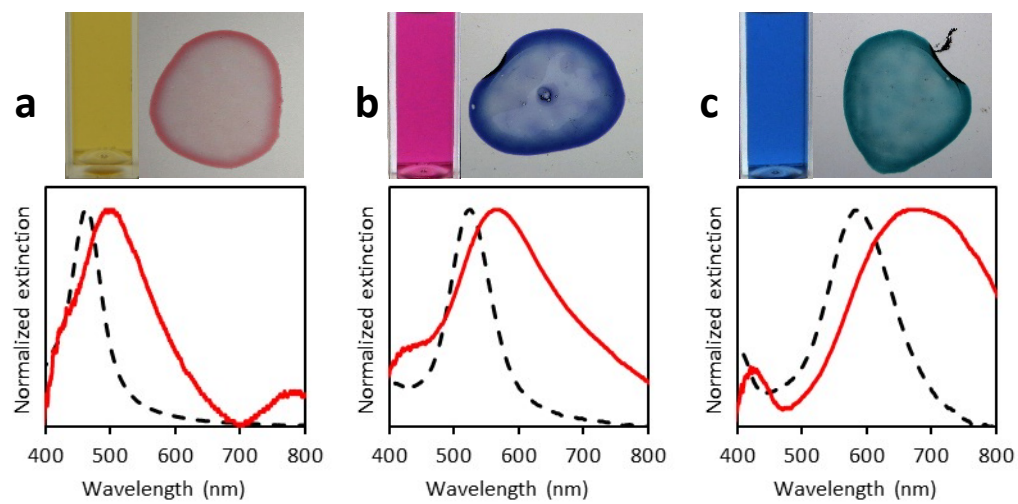

Figure 3. Optical characteristics of solutions and films of silver nanoparticles prepared with ascorbic acid. Top are the photographs of solution and film of (a) yellow, (b) red, and (c) blue colors. The bottom are the extinction spectra of (broken black) solutions and (solid red) films

Shape-tuned and size-tuned syntheses were similar in that both produced solutions of primary colors: yellow, red and blue (Figure 2 and Figure 3). In general, all solutions produced transparent films of a different color from the color of the mother solution. Also, all films showed the well-known coffee-ring effect, where non-volatile solutes concentrate at the edge of a drop that dries on a surface, thus the color is more intense at the edge of the film. In contrast, shape-tuned and size-tuned syntheses were different in the appearances (shade, homogeneity and color) of the films made. Shape-tuned nanoparticles produced darker (a color that looks more black), less intense, and less homogeneous films than size-tuned nanoparticles. Furthermore, the blue solution from shape-tuned nanoparticles produced a film with distinctive yellow, purple, and blue zones (Figure 2c).
These results demonstrate that films of various colors, and not just yellow, can be prepared by blending premade nanoparticles in a PVA matrix. There are two major observations from these syntheses. The first one is that the color discrepancy between solution and film was similar for both shape-tuned and size-tuned syntheses. The second is that the appearance of films depended on the physical property, size or shape, used to tune the color of the solution. It is well known that the Localized Surface Plasmon Resonance (LSPR) of silver nanoparticles depends on the shape and size of nanoparticles [32-35], as well as, on the environment that surrounds the nanoparticle [36]. Since the color observed from nanoparticles is the combination of the light that is not absorbed by the nanoparticle's LSPR, color changes may indicate morphological changes of the nanoparticle during the production of the film or just environmental changes.

Table 1. Summary of optical characteristics of solutions and films. The LSPR is the average of at least three trials, and the size is the average of at least 50 particles

\begin{tabular}{|c|c|c|c|c|c|c|}
\hline & & Solution & & & Film & \\
\hline & Color & LSPR (nm) & Size $(\mathrm{nm})$ & Color & LSPR $(\mathrm{nm})$ & Size (nm) \\
\hline \multirow{5}{*}{ 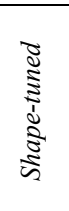 } & Yellow & $405 \pm 2$ & $2.4 \pm 0.5$ & Yellow & $430 \pm 8$ & $5.9 \pm 1.2$ \\
\hline & Red & $491 \pm 8$ & $29 \pm 11$ & Red & $510 \pm 20$ & $25 \pm 11$ \\
\hline & \multirow{3}{*}{ Blue } & \multirow{3}{*}{$620 \pm 40$} & \multirow{3}{*}{$\begin{array}{c}5.2 \pm 1.2 \\
19 \pm 5 \\
35 \pm 10\end{array}$} & Yellow zone & $449 \pm 5$ & $19 \pm 7$ \\
\hline & & & & Purple zone & $539 \pm 9$ & $20 \pm 4$ \\
\hline & & & & Blue zone & $680 \pm 70$ & $\begin{array}{l}59 \pm 15 \\
32+13\end{array}$ \\
\hline \multirow{3}{*}{ 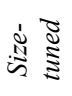 } & Yellow & $456 \pm 10$ & $16 \pm 6$ & Red & $490 \pm 20$ & $18 \pm 5$ \\
\hline & Red & $516 \pm 8$ & $26 \pm 7$ & Purple & $556 \pm 11$ & $26 \pm 9$ \\
\hline & Blue & $629 \pm 6$ & $29 \pm 10$ & Turquoise & $715 \pm 10$ & $29 \pm 11$ \\
\hline
\end{tabular}


As summarized in Table 1, the LSPR of all nanoparticles shifted towards longer wavelengths when nanoparticles were embedded in the PVA film. Yellow solution from shape-tuned nanoparticles (Figure 2a) and corresponding film had LSPRs in the violet region of the spectra (380 to $450 \mathrm{~nm}$ ), thus both solution and films appeared yellow. In contrast, the LSPR of the yellow solution from size-tuned nanoparticles was in the violet region, but the LSPR of the corresponding films was in the cyan region (490 to $520 \mathrm{~nm}$ ); since the LSPR of the film absorbed a different color, the film appeared red (Figure 3a). Red solutions from both syntheses had absorbances in the cyan region. Shape-tuned nanoparticles produced a red-purple film from a red solution (Figure 2b) because the absorbance of the film remained in the cyan region. On the contrary, size-tuned nanoparticle produced a purple film from a red solution (Figure $3 b$ ) because the absorbance of the film felt in the green region (520 to 560 $\mathrm{nm})$. Nanoparticles in both of the blue solutions had LSPRs in the red region of the spectra (620 to $750 \mathrm{~nm}$ ) and produced films with LSPR in the red region, but sizetuned nanoparticles produced a film with LSPR closer to the upper edge of the spectra that appeared turquoise in color (Figure 3c). The magnitude of the shifts was larger for LSPRs closer to the infrared; for instance, size-tuned nanoparticles had shift of $+34 \mathrm{~nm}$ for the yellow solution but $+86 \mathrm{~nm}$ for the blue solution. This behavior of the LSPR has been predicted as a response to changes in the refractive index of the media surrounding the nanoparticle [37]. Changes in refractive index is a plausible explanation for the color discrepancy between solution and film. Morphological changes of the nanoparticles is another possibility that can contribute to both the color discrepancy from solution to film and the difference in appearance between the films made by tuning either the size or shape of nanoparticles.

\subsection{Optical Stability during Preparation of Films}

During the preparation of the films, the change of color depended on the method used to prepare the nanoparticles. Figure 4 contrasts the LSPR of the mother solution (initial LSPR) to the LSPR after each of the three steps: centrifugation, dilution in PVA, and solvent evaporation. Each point in the graphs (Figure $4 a, 4 b$, and $4 c$ ) represents a single experiment. These values are replicas of the same condition to obtain yellow, red, and blue solutions as described in the Methods section or variations of these conditions to obtain solutions of other colors such as orange or purple. A diagonal line was added to each graph to aid in identifying the direction of the color change. For instance, points that fall above the diagonal line mean that the step caused the LSPR to shift towards longer wavelengths.

Centrifugation and dispersion of nanoparticles in water did not significantly shift the LSPR for size-tuned nanoparticles (Figure 4a). Centrifugation both reduces the volume of solvent and facilitates the interaction of nanoparticles [38]. For nanoparticles that are in contact or very close proximity, the coupling of their LSPRs results in a large LSPR shift towards long wavelengths [39]. The lack of such large LSPR shift suggests that the presence of
PVP prevented nanoparticles from aggregating. In contrast, the LSPR for shape-tuned nanoparticles shifted towards shorter wavelengths upon centrifugation and dispersion in water (Figure 4a). Similar shifts observed in previous research were attributed to direct and indirect effects of centrifugation on shape-tuned nanoparticles. A direct effect of centrifugation was the removal of reagents before complete growth of nanoparticles [40]. An indirect effect of centrifugation was introducing oxygen when pellets were dispersed in water causing nanoparticles to reshape into more isotropic structures [41].

Dilution in PVA shifted the LSPR of both shape-tuned and size-tuned nanoparticles only very slightly $(\sim 5 \mathrm{~nm})$ towards longer wavelengths (Figure 4b). By introducing PVA in solution, the refractive index surrounding the nanoparticles increased causing the LSPR to shift towards longer wavelengths [36]. The small magnitude of the shift towards longer wavelengths does not indicate aggregation of nanoparticles as shifts caused by aggregation are expected on a larger magnitude [39]. For shape-tuned nanoparticles, dissolution in PVA did not counterbalance the shift caused by centrifugation; thus the LSPR of nanoparticles in the film remained at shorter wavelengths than the LSPR of nanoparticles in the mother solution (Figure 4b). It was previously reported that in order to entrap colored nanoparticles in PVA polymer, solid PVA was dissolved at $80{ }^{\circ} \mathrm{C}$ in a yellow solution of silver nanoparticles for several hours [20]. However, heating resulted in loss of color as PVP does not provide protection from thermal reshaping of nanoparticles [41]. In this work, rather than dissolving solid PVA in the nanoparticle solution, concentrated PVA was diluted with silver nanoparticles and mixed. Dilution preserved the color of the solution and decreased synthesis time.

For size-tuned nanoparticles, evaporation of water caused a LSPR shift towards longer wavelengths (Figure 4c). As shown in equation (1), the magnitude of the shift increases as the LSPR moves towards longer wavelengths.

$$
\left(\frac{\Delta L S P R_{\text {film }}}{\Delta L S P R_{\text {solution }}}\right)=1.4
$$

It has been calculated that increasing the refractive index of the media causes larger LSPR shift for nanoparticles with light extinction closer to the infrared [37]. In this work, nanoparticles experience an increase of refractive index from water (1.33) to PVA (1.52) [42]. Aggregation of nanoparticles could also cause a large LSPR shift [39]; however, aggregates were not observed in the TEM images (vide infra) for any of the nanoparticles. Shape-tuned nanoparticles also had a larger LSPR shift towards longer wavelengths upon water evaporation than upon dilution in PVA. However, because films prepared from shape-tuned nanoparticles showed different colored zones (Figure $2 b$ and $2 c$ ), several values of LSPR were found at shorter and longer wavelengths than the LSPR in the mother solution; thus, a correlation of shifts, as in equation 1, cannot be made for shape-tuned nanoparticles. The segregation of colors in the films from shape-tuned nanoparticles, but not from size-tuned nanoparticles, suggests that the shape of the nanoparticle played a determining role in the distribution of particles during the evaporation of the solvent. 

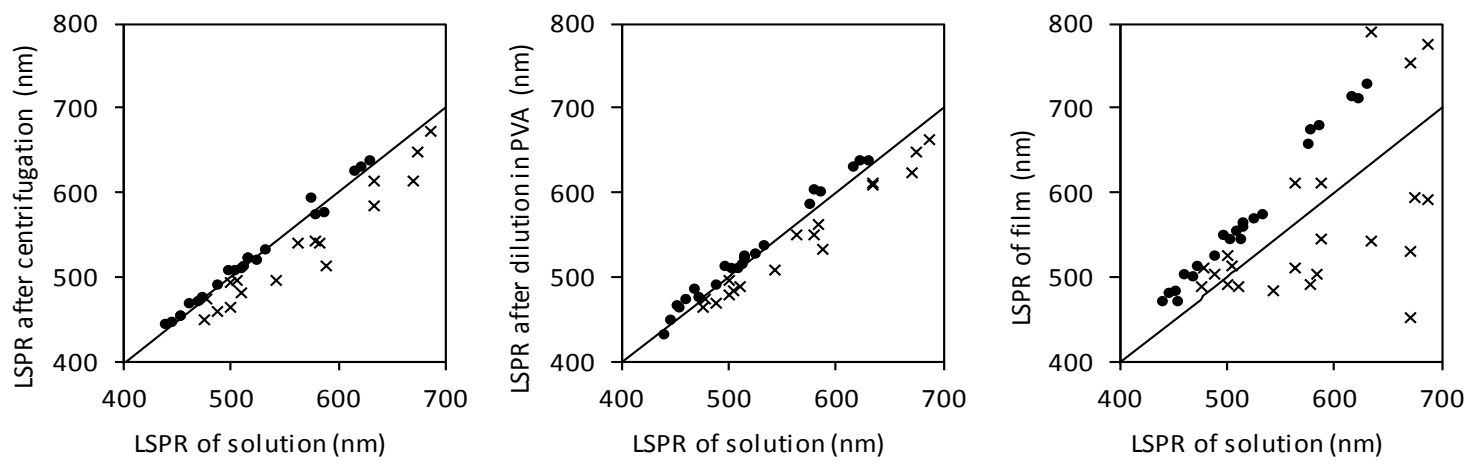

Figure 4. Changes in LSPR of $(\mathrm{x})$ shape-tuned and $(\bullet)$ size-tuned nanoparticles during (a) centrifugation, (b) dilution in PVA, and (c) solvent evaporation

It is known that a larger LSPR sensitivity can be achieved by modifying the shape of nanoparticles in solutions rather than their size. This morphology effect does not seem to be as advantageous on films. Different size-tuned nanoparticles had similar changes during the preparation of the films. On the contrary, changes from shape-tuned nanoparticles are scattered and their magnitudes do not correlate to the LSPR of nanoparticles in solution. All these results suggest that shape-tuned nanoparticles are less resistant to environmental changes and have a greater influence on the color distribution of the film than size-tuned nanoparticles.

\subsection{Correlation between Appearance of the Films and Nanoparticle's Morphology}

Since the appearance of the films depends on the method used to synthesize the nanoparticles, TEM was used to investigate the morphology of the nanoparticles in solutions and films prepared from shape-tuned (Figure 5) and size-tuned nanoparticles (Figures 6). The sizes, diameters for nanosphere and nanodisk or side length for nanoprims, are summarized in Table 1.

Shape-tuned nanoparticles became less isotropic as the LSPR moved toward longer wavelengths. Similar to previous reports using citrate and borohydride to tune the shape of silver nanoparticles [8,30], spheres, disks, and prisms were observed in yellow (Figure 5a), red (Figure 5b), and blue (Figure 5c) solutions, respectively.
Although, the blue solution contained a variety of nanoparticles of different shapes and sizes, the prevailing color was blue; this observation agrees with previous studies showing that the extinction coefficient of nanoparticles increases as the LSPR moves towards larger wavelengths [43] and decreases for isotopic nanoparticles [44]. Films obtained from shape-tuned nanoparticles had nanoparticles of similar sizes and shapes than their mother solution. Since the film from the blue solution had different colored zones (Figure 2c), micrographs were taken from each zone of the blue film. Figures $5 \mathrm{f}$ to $5 \mathrm{~h}$ show that the yellow and purple zones had small disks. While, the blue zone had nanodisks (diameter $=32 \mathrm{mn}$ ) and larger nanoprims (length $=59 \mathrm{~nm}$ ) with rounded tips than the nanoprims in the mother solution (length $=35$ $\mathrm{nm}$ ). Since increasing the isotropy of nanoparticles should result in LSPR shifting towards shorter wavelength [35], it is possible that the rounding of the tips of nanoprims occurred during the centrifugation step (Figure 4a). Tang and coworkers reported similar rounding of the tips of nanoprims upon centrifugation and dispersion in water [41]. They also reported that reshaping nanodisks back into nanoprims produced nanoprims of larger sizes. Increasing the size of nanoprims should result in a LSPR shifted towards longer wavelengths. As summarized in Table 1, the blue film from shape-tuned nanoparticles had LSPRs at both shorter (449 and $539 \mathrm{~nm})$ and longer $(680 \mathrm{~nm})$ wavelengths than the LSPR of the mother solution $(620 \mathrm{~nm})$.
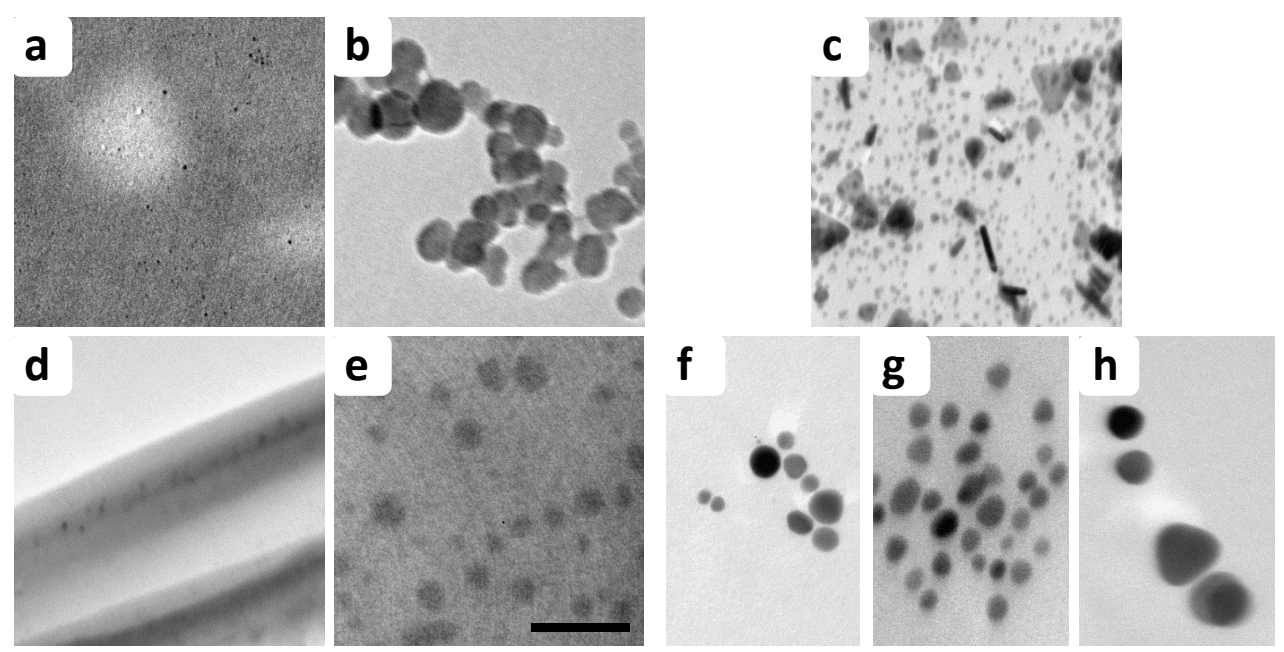

Figure 5. TEM of (top) solutions and (bottom) films from shape-tuned nanoparticles. (a, d) Yellow and (b, e) red films and solutions. (c) Blue solution, and (f) yellow, (g) purple, and (h) blue zones, respectively, of film produced from blue solution. Scale bar = $100 \mathrm{~nm}$ and applies for all micrographs 


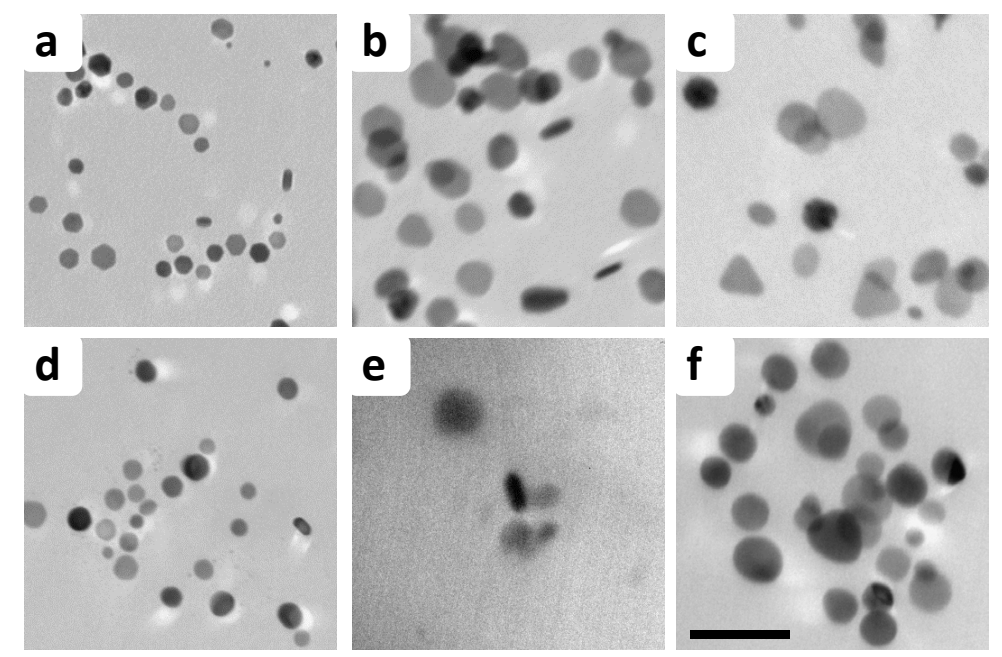

Figure 6. TEM of (a to c) solutions and (d to f) films prepared with ascorbic acid. (a, d) Yellow, (b, e) red, and (c, f) blue. Scale bar = 100 nm and applies for all micrographs

Figure 6 shows that solutions from size-tuned nanoparticles had mostly nanodisks, and the diameter of the nanodisks increased as the color moved from yellow (Figure 6a) to red (Figure 6b) to blue (Figure 6c). Some triangles were also found in the blue solution. The corresponding films exhibited a similar trend in size (Figure 6d to 6f). These findings agree with Mahmoud's work, in which nanodisk of fixed thicknesses and increasing diameters were prepared by decreasing the amount of ascorbic acid [31]. The red solution from shape-tuned nanoparticles $(\mathrm{LSPR}=491 \mathrm{~nm})$ and the blue solution from size-tuned nanoparticles $(\mathrm{LSPR}=629 \mathrm{~nm})$ had nanodisks of the same diameter, $29 \mathrm{~nm}$. The fact that these nanoparticles had very different LSPRs suggests that these nanodisks had different thicknesses. Given that thinner nanodisks have LSPR at longer wavelengths than thicker nanodisks with the same diameter [31], it is likely that the size-tuned synthesis produced blue solutions with thinner nanodisks than the nanodisks in the red solution prepared by controlling the shape of nanoparticles. Table 1 shows that the sizes of nanoparticles in solution and corresponding film were within one standard deviation, except for the smaller nanoparticles $(2.4 \mathrm{~nm}$ and $5 \mathrm{~nm})$. This last discrepancy was attributed to the intrinsic uncertainty associated with the preparation of the films. For instance, ultramicrotomed sections from the same film may have different distribution of nanoparticles at different depths [45], and the embedding media causes micrographs of nanoparticles in films to be less clear than the micrographs of nanoparticles in solution. This uncertainty favors the measurement of large nanoparticles. In spite of these uncertainties, TEM images confirmed that only the most anisotropic nanoparticles (prims) showed a slight difference between solution and film.

All solutions that produced films of a single color had a single composition of nanoparticle in both solution and film. On the contrary, the blue solution from shape-tuned nanoparticles had a heterogeneous composition of nanoparticles (Figure 5c) and therefore produced a film with various colored zones (Figure 2c). The micrographs (Figure 5f, 5g, and 5h) shows that these zones contained nanoparticles of different shapes and sizes. Such segregation has been previously observed for bidispersed systems, where nanoparticles of different sizes segregate into zones with particles of a single size and zones with particles of mixed sizes [46]. Moreover, it has been predicted that shape of nanoparticle plays a larger role in the segregation of particles in films due to difference in attractive depletion, especially for nanoparticles with flat faces [45].

\section{Conclusions}

Tuning the optical properties of silver nanoparticles in solution has been widely attempted by tuning the shape, rather than the size of nanoparticles, because shape control result in more sensitive LSPRs than size control. On the contrary, it is more appropriate to use size-tuned nanoparticles than shape-tuned nanoparticles when tuning the color of films. Phenomena intrinsic to the formation of films, like the coffee-ring effect and particle segregation, and the homogeneity of the nanoparticles in solution become relevant; consequently, size-tuned nanoparticles produce more homogeneous and intensely colored films. This work presents a simple approach to prepare colored nanocomposite films that preserved the morphologies of silver nanoparticles. This approach can potentially be used to study bidispersed systems and to effectively tune the optical properties of nanocomposite films, thus aiding in the design of chemical sensors.

\section{Acknowledgments}

This work was support by Skidmore College faculty development program and Skidmore's Summer Collaborative Research Program. The authors thank Lisa Quimby and Juan Navea for useful discussion and comments. Thanks to Lily Sun and David Domozych from the Skidmore College Microscopy Center (SMIC) for training and use of TEM and Ultramicrotome.

\section{Statement of Competing Interests}

The authors declare that there is no conflict of interest regarding the publication of this paper. 


\section{References}

[1] Hsu, S-W., Rodarte, A.L., Som, M., Arya, G., and Tao, A.R., "Colloidal Plasmonic Nanocomposites: From Fabrication to Optical Function", Chemical Review, 118 (6) 3100-20. 2018.

[2] Prakash, J., Harris, R.A., and Swart, H.C, "Embedded plasmonic nanostructures: synthesis, fundamental aspects and their surface enhanced Raman scattering applications", International Reviews in Physical Chemistry, 35 (3) 353-98. 2016.

[3] Abargues, R., Rodriguez-Canto, P.J., Albert, S., Suarez, I., and Martinez-Pastor, J.P., "Plasmonic optical sensors printed from Ag-PVA nanoinks", Journal of Material Chemistry C, 2 (5) 90815. 2014.

[4] Ramesh, G.V., and Radhakrishnan, T.P., "A Universal Sensor for Mercury (Hg, Hg-I, Hg-II) Based on Silver NanoparticleEmbedded Polymer Thin Film", ACS Applied Materials \& Interfaces, 3 (4) 988-94. 2011.

[5] Nimrodh-Ananth, A., Umapathy, S., Sophia, J., Mathavan, T., and Mangalaraj, D., "On the optical and thermal properties of in situ/ex situ reduced Ag NP's/PVA composites and its role as a simple SPR-based protein sensor", Applied Nanoscience, 1 (2) 87-96. 2011.

[6] Hariprasad, E., and Radhakrishnan, T.P. "In Situ Fabricated Polymer-Silver Nanocomposite Thin Film as an Inexpensive and Efficient Substrate for Surface-Enhanced Raman Scattering", Langmuir, 29 (42) 13050-7. 2013

[7] Yu, D.G., Lin, W.C., Lin, C.H., Chang, L.M., and Yang, M.C., "An in situ reduction method for preparing silver/poly(vinyl alcohol) nanocomposite as surface-enhanced Raman scattering (SERS)-active substrates", Materials Chemistry Physics, 101 (1) 93-8. 2007

[8] Huang, T., and $\mathrm{Xu}, \mathrm{X} . \mathrm{H} . \mathrm{N}$., "Synthesis and characterization of tunable rainbow colored colloidal silver nanoparticles using single-nanoparticle plasmonic microscopy and spectroscopy" Journal of Materials Chemistry, 20 (44) 9867-76. 2010.

[9] Mendis, P., de Silva, R.M., de Silva, K.M.N., Wijenayaka, L.A., Jayawardana, K., and Yan, M., "Nanosilver rainbow: a rapid and facile method to tune different colours of nanosilver through the controlled synthesis of stable spherical silver nanoparticles" $R S C$ Advances, 6 (54) 48792-9. 2016.

[10] Rivero, P.J., Goicoechea, J., Urrutia, A., Matias, I.R., and Arregui, F.J., "Multicolor Layer-by-Layer films using weak polyelectrolyte assisted synthesis of silver nanoparticles", Nanoscale Research Letters, 8, 1-9. 2013.

[11] De, G., Medda, S.K., De, S., and Pal, S., "Metal nanoparticle doped coloured coatings on glasses and plastics through tuning of surface plasmon band position", Bulletin of Materials Science, 31 (3) 477-85. 2008

[12] Boev, V.I., Perez-Juste, J., Pastoriza-Santos, I., Silva, C.J.R., De Gomes, M., and Liz-Marzan, L.M., "Flexible Ureasil Hybrids with Tailored Optical Properties through Doping with Metal Nanoparticles", Langmuir, 20 (23) 10268-72. 2004.

[13] Caseri, W., "Color switching in nanocomposites comprising inorganic nanoparticles dispersed in a polymer matrix", Journal of Materials Chemistry, 20 (27) 5582-92. 2010.

[14] Liz-Marzan, L.M., "Tailoring Surface Plasmons through the Morphology and Assembly of Metal Nanoparticles", Langmuir, 22 (1) 32-41. 2006.

[15] Wilson, O., Wilson, G.J., and Mulvaney, P., "Laser writing in polarized silver nanorod films", Advanced Materials, 14 (13-14) 1000-4. 2002.

[16] Liu, L., Gao, Z., Jiang, B., Bai, Y., Wang, W., and Yin, Y., "Reversible Assembly and Dynamic Plasmonic Tuning of Ag Nanoparticles Enabled by Limited Ligand Protection", Nano Letters, 18 (8) 5312-8. 2018.

[17] Ghanipour, M., and Dorranian, D., "Effect of Ag-nanoparticles doped in polyvinyl alcohol on the structural and optical properties of PVA films", Journal of Nanomaterials, 897043, 1-11. 2013.

[18] Saini, I., Rozra, J., Chandak, N., Aggarwal, S., Sharma, P.K., and Sharma, A., "Tailoring of electrical, optical and structural properties of PVA by addition of Ag nanoparticles", Materials Chemistry and Physics, 139 (2-3) 802-10. 2013

[19] Vodnik, V.V., Saponjic, Z., Dzunuzovic, J.V., Bogdanovic, U., Mitric, M., and Nedeljkovic, J., "Anisotropic silver nanoparticles as filler for the formation of hybrid nanocomposites", Materials Research Bulletin, 48 (1) 52-7. 2013.
[20] Duncan, K.A., et al. "Art as an Avenue to Science Literacy: Teaching Nanotechnology through Stained Glass", Journal of Chemical Education, 87 (10) 1031-8. 2010.

[21] Porel, S., Singh, S., Harsha, S.S., Rao, D.N., and Radhakrishnan, T.P., "Nanoparticle-embedded polymer: In situ synthesis, freestanding films with highly monodisperse silver nanoparticles and optical limiting", Chemistry of Materials, 17 (1) 9-12. 2005.

[22] Feng, Q.Q., Dang, Z.M., Li, N., and Cao, X.L., "Preparation and dielectric property of Ag-PVA nano-composite", Materials Science and Engineering B-Solid State Materials for Advanced Technology, 99 (1-3) 325-8. 2003.

[23] Mbhele, Z.H., Salemane, M.G., van Sittert, C., Nedeljkovic, J.M., Djokovic, V., and Luyt, A.S., "Fabrication and characterization of silver-polyvinyl alcohol nanocomposites", Chemistry of Materials, 15 (26) 5019-24. 2003.

[24] Ramesh, G.V., Porel, S., and Radhakrishnan, T.P., "Polymer thin films embedded with in situ grown metal nanoparticles", Chemical Society Reviews, 38 (9) 2646-56. 2009.

[25] Prakash, J., Pivin, J.C., and Swart, H.C., "Noble metal nanoparticles embedding into polymeric materials: From fundamentals to applications", Advances in Colloidal and Interface Sciences, 226 (Part B) 187-202. 2015.

[26] Abargues, R., Marques-Hueso, J., Canet-Ferrer, J., Pedrueza, E. Valdes, J.L., Jimenez, E., and Martinez-Pastor, J.P., "Highresolution electron-beam patternable nanocomposite containing metal nanoparticles for plasmonics", Nanotechnology, 19 (35) 355308. 2008

[27] Tyurin, A., De Filpo, G., Cupelli, D., Nicoletta, F.P., Mashin, A., and Chidichimo, G., "Particle size tuning in silverpolyacrylonitrile nanocomposites", eXPRESS Polymer Letters, 4 (2) 71-8. 2010.

[28] Hore, M.J.A., Frischknecht, A.L., and Composto, R.J.,"Nanorod Assemblies in Polymer Films and Their Dispersion-Dependent Optical Properties", ACS Macro Letters, 1 (1) 115-21. 2012.

[29] Metraux, G.S., and Mirkin, C.A., "Rapid thermal synthesis of silver nanoprisms with chemically tailorable thickness", Advanced Materials, 17 (4) 412-5. 2005.

[30] Tang, B., Li, J.L., Hou, X.L., Afrin, T., Sun, L., and Wang, X.G., "Colorful and Antibacterial Silk Fiber from Anisotropic Silver Nanoparticles", Industrial \& Engineering Chemistry Research, 52 (12) 4556-63. 2013

[31] Mahmoud, M.A., "Simultaneous Reduction of Metal Ions by Multiple Reducing Agents Initiates the Asymmetric Growth of Metallic Nanocrystals", Crystal Growth \& Design, 15 (9) 4279-86. 2015.

[32] Haes, A.J., Haynes, C.L., McFarland, A.D., Schatz, G.C., Van Duyne, R.R., and Zou, S.L., "Plasmonic materials for surfaceenhanced sensing and spectroscopy", MRS Bulletin, 30 (5) 368-75. 2005.

[33] Lee, K.S., and El-Sayed, M.A., "Gold and silver nanoparticles in sensing and imaging: Sensitivity of plasmon response to size, shape, and metal composition", Journal of Physical Chemistry B, 110 (39) 19220-5. 2006

[34] Mulvaney, P., "Surface plasmon spectroscopy of nanosized metal particles", Langmuir, 12 (3) 788-800. 1996.

[35] Kelly, K.L., Coronado, E., Zhao, L.L., and Schatz, G.C., "The optical properties of metal nanoparticles: The influence of size, shape, and dielectric environment", Journal of Physical Chemistry B, 107 (3) 668-77. 2003.

[36] Pelton, M., and Bryant, G.W., Introduction to Metal-Nanoparticle Plasmonics, WILEY, 2013.

[37] Haes, A.J., Zou, S., Schatz, G.C., and Van Duyne, R.P., "Nanoscale Optical Biosensor: Short Range Distance Dependence of the Localized Surface Plasmon Resonance of Noble Metal Nanoparticles", Journal of Physical Chemistry B, 108 (22) 69616968. 2004

[38] Roca, M., Pandya, N., Nath, S., and Haes, A., "Linear Assembly of Gold Nanoparticle Clusters via Centrifugation", Langmuir, 26 (3) 2035-41. 2010

[39] Ghosh, S.K., and Pal, T., "Interparticle Coupling Effect on the Surface Plasmon Resonance of Gold Nanoparticles: From Theory to Applications", Chemical Reviews, 107 (11) 4797-862. 2007.

[40] Shi, W., Casas, J., Venkataramasubramani, M., and Tang, L., "Synthesis and characterization of gold nanoparticles with plasmon absorbance wavelength tunable from visible to near infrared region", ISRN Nanomaterials, 659043, 1-10. 2012. 
[41] Tang, B., Xu, S.P., Hou, X.L., Li, J.H., Sun, L., Xu, W,Q., and Wang, X.G., "Shape Evolution of Silver Nanoplates through Heating and Photoinduction", ACS Applied Materials \& Interfaces, 5 (3) 646-53. 2013.

[42] Mahmoud, K.H., "Synthesis, characterization, optical and antimicrobial studies of polyvinyl alcohol-silver nanocomposites", Spectrochimica Acta, Part A, 138, 434-40. 2015.

[43] Haiss, W., Thanh, N., Aveyard, J., and Fernig, D., "Determination of size and concentration of gold nanoparticles from UV-Vis spectra", Analytical Chemistry, 79 (11) 4215-21. 2007.
[44] Mock, J.J., Barbic, M., Smith, D.R., Schultz, D.A., and Schultz, S., "Shape effects in plasmon resonance of individual colloidal silver nanoparticles", Journal of Chemical Physics, 116 (15) 6755-9. 2002.

[45] Teng, C.Y., Sheng, Y.J., and Tsao, H.K., "Particle size-induced transition between surface segregation and bulk aggregation in a thin film of athermal polymer-nanoparticle blends", Journal of Chemical Physics, 146 (1) 014904. 2017.

[46] Patil, N.D., Bhardwaj, R., and Sharma, A., "Self-Sorting of Bidispersed Colloidal Particles Near Contact Line of an Evaporating Sessile Droplet", Langmuir, 34 (40) 12058-70. 2018.

(C) The Author(s) 2019. This article is an open access article distributed under the terms and conditions of the Creative Commons Attribution (CC BY) license (http://creativecommons.org/licenses/by/4.0/). 